\title{
A Case Report of an Unusual Presentation: Could the Early Use of Interventional Radiology have Saved this Patient of Scrub Typhus
}

Habib Md Reazaul Karim¹, Prithwis Bhattacharyya ${ }^{2}$, Md. Yunus ${ }^{3}$

\begin{abstract}
A 19-year-old soldier was referred to our Intensive Care Unit (ICU) with a diagnosis of pyrexia of unknown origin in a state of deep coma (GCS 6/15) and shock with severe anaemia at presentation without any evidence of external or internal bleeding. He was subsequently diagnosed as a case of Scrub Typhus with Multi Organ Dysfunction (MODS). Clinical evidence of massive Gastrointestinal (GI) bleeding (melaena and clotted blood per rectum) was found next day. Despite aggressive supportive treatment we lost the patient next morning. We are presenting the case to share our experience for better management of such cases in future along with a point to ponder which probably would give an opportunity to critical care physicians to save such patients.
\end{abstract}

Keywords: Scrub Typhus, Acute GI bleed, Melaena. Interventional radiology.

\section{Introduction}

Clinical presentations of scrub typhus are non-specific. About $22.7 \%$ of the Scrub Typhus patients have gastro intestinal manifestations and all of the symptomatic patients have one or more endoscopic abnormalities ${ }^{1}$. Though GI manifestations are common, massive bleeding is rare and if appropriate and early multidisciplinary actions are not taken, the patient may end up with fatality.

\section{Case}

A 19 year-old soldier was referred from a Military Hospital $(\mathrm{MH})$ with a history of fever for 10 days along with altered sensorium, severe anaemia with coagulopathy. He was shifted to our Intensive Care Unit (ICU) in view of deterioration of consciousness and was shifted with AMBU and mask ventilation with a GCS of E2V1M3. He was intubated immediately and put on Mechanical Ventilator. Two units Packed Red Blood Cells (PRBC) were transfused in MH. CSF analysis, peripheral blood smear for malaria parasite, Paracheck, Widal tests, HBsAg, Anti HCV and

1. Dr. Habib Md Reazaul Karim

Senior Resident and Critical Care Medicine Trainee. Department of Anaesthesiology and Critical Care.

2. Dr. Prithwis Bhattacharyya

Professor \& HOD. Department of Anaesthesiology and Critical Care.

3. Dr. Md. Yunus

Professor. Department of Anaesthesiology and Critical Care.

All the authors work in North Eastern Indira Gandhi Regional Institute of Health and Medical Science, Shillong, Meghalaya, India.

\section{Corresponding Author}

Dr. Prithwis Bhattacharyya

Professor and Head

Department of Anaesthesiology and Critical Care.

NEIGRIHMS,

Shillong. Meghalaya, India 793018.

E mail: prithwisbhat123@gmail.com
Dengu were found to be negative. Patient was on Inj. Artesunate, Inj.Acyclovir and Inj. Ceftriaxone in the MH.

The Patient was in shock with blood pressure (BP) not recordable along with metabolic acidosis of $6.9 \mathrm{pH}$. There were no external signs of bleeding. One $16 \mathrm{G}$ intravenous cannula was placed and USG guided Central venous line inserted. Fluid resuscitation was started with crystalloid along with PRBC. Despite adequate CVP, BP was low along with very low systemic vascular resistance (SVR). Inj. Noradrenaline was started and titrated to MAP of $>/=$ $70 \mathrm{mmHg}$.

Clinically, differential diagnosis of Scrub Typhus or Leptospirosis with MODS including ARDS was made. Empiric Tab. Doxycycline through Ryle's tube was added to Injection Ceftriaxone. Inj. Artesunate continued while Inj. Acyclovir was stopped as viral infection was thought to be unlikely.

Liver function tests revealed enzymes grossly raised (except serum bilirubin). Other positive findings were $\mathrm{Hb}-4.6 \mathrm{gm} \%$, INR 5.66, Potassium 5.9 meq/L, Serum Creatinine $2.1 \mathrm{mg} / \mathrm{dl}$, TLC $17,700 / \mathrm{dl}$ (with $75 \%$ neutrophils) with no toxic granules or band forms. Platelet count was normal. Chest X-ray revealed homogeneous opacity over all the four quadrants. $\mathrm{PaO}_{2} / \mathrm{FiO}_{2}$ found to be $<100$.

Three units of PRBC, 5 units of Fresh Frozen Plasma (FFP) were transfused on the first day. Tapering of Vasopressor support was started once becomes hemodynamically stable. $\mathrm{Hb}$ improved to $8 \mathrm{gm} \%$.

Next morning 2 episodes of melaena (large quantity) were noted but Ryle's tube aspirate showed no blood. Infusion Pantoprazole was started. Hb was falling again (6gm \%) and the patient was found to be heading towards haemodynamic collapse. At 9.00 a.m. approximately 3 fist sized clots were noted in stool and $\mathrm{Hb}$ came to $4 \mathrm{gm} \%$ despite ongoing PRBC transfusion. Immediate surgical referral was given and proctoscopy found to be normal. Bedside USG revealed hepatomegaly with minimal ascitic fluid. Bedside 
Echocardiography revealed poor LV function. Invasive haemodynamic monitoring using EV1000 Vigileo monitor of Edwards Lifesciences revealed low cardiac output (CO), SVR and stroke volume (SV). Inj. Dobutamine was added to the already running Noradrenaline infusion.

Interventional radiology referral was given for CT Angiogram along with request to assess feasibility of embolization of feeding vessels if any. Feasibility of exploratory laparotomy was also discussed with surgical team. They were reluctant to explore surgically in view of patient's condition and wanted CT angiogram report before proceeding.

At 12 Noon patients report for scrub typhus (Weil-felix test and $\operatorname{IgM}$ ) came positive. Inj. Azithromycin was added over and above the ongoing regimen. Patient received 3 more units of PRBC and 4 units of FFP. Despite ongoing blood transfusion, $\mathrm{Hb}$ rose only minimally ( $\mathrm{Hb} 5 \mathrm{gm} \mathrm{\% )}$ and patient remained on ventilator, Vasopressor + Ionotropic support. Extra Vascular Lung Water (EVLW) was found to be $>12 \mathrm{ml} / \mathrm{kg}$ and ventilation as per ARDS.net protocol along with other support.

Despite all these steps, the patient's condition continued to deteriorate. Two more units of PRBC transfused immediately and demand for more units of blood components sent but patient went to cardiac arrest (possibly because of continued GI bleeding) and we lost him.

\section{Discussion}

Scrub Typhus leads to the destruction of endothelial cells and perivascular inflammation causing focal or disseminated vasculitis. $^{2}$ Systemic vasculitis of GI tract leads to GI symptoms and bleeding. Though GI symptoms occur in approximately $22.7 \%$ patients of Scrub Typhus, ${ }^{1}$ clinical GI Bleeding incidence is relatively uncommon ranging from $1.9 \%$ to $3.4 \%{ }^{1,3}$ Scrub Typhus is prevalent in India and currently there appears to be a resurgence. ${ }^{4}$ Though most of the infections are mild, about $13.8 \%$ cases land up with at least one complication. ${ }^{3}$

Diagnosis of scrub typhus is not always easy. Eschar and lymphadenopathy though regarded as pathognomonic are found in only $4 \%$ to $13.3 \%$ of Indian patients. ${ }^{5,6}$ Symptoms are very vague and suggest a wide range of differential diagnosis. Serological tests, though relatively cheap, are not widely available. Moreover, all currently available serological tests for scrub typhus have limitations. ${ }^{7}$ On the other hand, even newer strains of orientia tsutsugamushi are being reported.$^{8,9}$ All these factors lead to delayed diagnosis and it remains as undifferentiated fever if not suspected and investigated early in the course of disease. Delayed diagnosis by even 2 days leads to increased complications. ${ }^{3}$

Acute GI bleeding in scrub typhus can be life threatening and may even require surgery. ${ }^{10}$ In our case, site of the GI bleeding was not pin pointed and confirmation was awaited. Surgery is also risky in the face of severe coagulopathy and probably because of this; our surgical team was hesitant to proceed with exploratory laparotomy. Considering these points, therefore, interventional radiology would have given an advantage of early diagnosis and localization as well as therapeutic embolization. Unfortunately before we could proceed for the same we lost the patient.

\section{Conclusion}

Scrub typhus should be ruled out in all suspected tropical fever cases since; delaying treatment leads to various complications leading to death. Acute GI Bleeding should be suspected in refractory and clinically disproportionate anaemia and must be regarded as emergency. Multi disciplinary approach including interventional radiology should be sought at the earliest as a probable life saving procedure.

\section{Conflict of interest: None}

\section{Reference}

1. Kim SJ, Chung IK, Chung IS, Song DH, Park SH, Kim HS et al. The clinical significance of upper gastrointestinal endoscopy in gastrointestinal vasculitis related to scrub typhus. Endoscopy 2000;32(12):950-5.

2. Jeong YJ, Kim S, Wook YD, Lee JW, Kim KI, Lee SH. Scrub typhus: clinical, pathologic, and imaging findings. Radiographics 2007;27(1):161-72.

3. Yasunaga H, Horiguchi H, Kuwabara K, Hashimoto H, Matsuda S. Delay in tetracycline treatment increases the risk of complications in Tsutsugamushi disease: data from the Japanese Diagnosis Procedure Combination database. Intern Med 2011;50(1):37-42.

4. Chaudhry D, Goyal S. Scrub Typhus-resurgence of a forgotten killer. Indian J Anaesth 2013;57(2):135-6.

5. Girija S, Rajan A, Sathiyanarayanan J, Mangaiyarkarasi T, Saban P, Sunil S et al. Scrub typhus- An emerging disease in South India. IJRRMS 2013;3(4): 11-3.

6. Narvencar KPS, Rodrigues S, Nevrekar RP, Dias L, Dias A, Vaz M et al. Scrub typhus in patients reporting with acute febrile illness at a tertiary health care institution in Goa. Indian $\mathrm{J}$ Med Res 2012;136:1020-4.

7. Koh GC, Maude RJ, Paris DH, Newton PN, Blacksell SD. Review: Diagnosis of Scrub Typhus. Am. J. Trop. Med. Hyg 2010;82(3):368-70.

8. Yang HH, Huang IT, Lin CH, Chen TY, Chen LK. New Genotypes of Orientia tsutsugamushi Isolated from Humans in Eastern Taiwan. PLoS ONE 2012;7(10): e46997. doi:10.1371/journal.pone.0046997.

9. Yoo JR, Heo ST, Koh S, Kim S, Kim S. Unusual Genotypic Distribution of Orientia tsutsugamushi Strains Causing Human Infections on Jeju Island. Am J Trop Med Hyg 2014;90(3):507-10.

10. Bae KB, Youn WH, Lee YJ, Jung SJ, Hong KH. Massive small bowel bleeding caused by scrub typhus in Korea. World $\mathrm{J}$ Gastrointest Surg 2010;2(2):47-50. 